\title{
A robust blind medical image watermarking approach for telemedicine applications
}

\author{
Fares Kahlessenane $^{1} \cdot$ Amine Khaldi $^{1}$ (D) $\cdot$ Redouane Kafi $^{1} \cdot$ Salah Euschi $^{1}$
}

Received: 30 July 2020 / Revised: 14 November 2020 / Accepted: 23 November 2020/Published online: 10 February 2021

(C) Springer Science+Business Media, LLC, part of Springer Nature 2021

\begin{abstract}
In order to enhance the security of exchanged medical images in telemedicine, we propose in this paper a blind and robust approach for medical image protection. This approach consists in embedding patient information and image acquisition data in the image. This imperceptible integration must generate the least possible distortion. The watermarked image must present the same clinical reading as the original image. The proposed approach is applied in the frequency domain. For this purpose, four transforms were used: discrete wavelets transform, non-subsampled contourlet transform, non-subsampled shearlet transform and discreet cosine transform. All these transforms was combined with Schur decomposition and the watermark bits were integrated in the upper triangular matrix. To obtain a satisfactory compromise between robustness and imperceptibility, the integration was performed in the medium frequencies of the image. Imperceptibility and robustness experimental results shows that the proposed methods maintain a high quality of watermarked images and are remarkably robust against several conventional attacks.
\end{abstract}

Keywords Medical image - Digital watermarking · Discrete wavelet transform · Non-subsampled contourlet transform . Non-subsampled shearlet transform $\cdot$ Discreet cosine transform $\cdot$ Schur decomposition

\section{Introduction}

The use of interfaces for remote medical data visualization is currently in high demand. These interfaces provide access to patient records containing text and image data [1]. This sharing of this data, and in particular images on the Internet, exposes them to unauthorized manipulation [2], despite existing security tools such as access control. Watermarking has therefore been proposed to increase the

Amine Khaldi

Khaldi.Amine@univ-ouargla.dz

Fares Kahlessenane

Kahlessnane.Fares@univ-ouargla.dz

Redouane Kafi

Kafi.Redouane@univ-ouargla.dz

Salah Euschi

Euchi.Salah@univ-ouargla.dz

1 Artificial Intelligence and Information Technology Laboratory (LINATI), Computer Science Department, Faculty of Sciences and Technology, University of Kasdi Merbah, 30000 Ouargla, Algeria sharing security by allowing keeping the patients' data confidential and verifying the medical images' integrity [3]. Researchers have been increasingly interested in this field and several techniques have already been proposed in this field. However, the medical image has its own specificities [4]. The watermarked image must present the same clinical reading as the original image, so it must not suffer any degradation that affects the diagnosis [5]. The image watermarking methods can be classified according to the insertion domain. Spatial methods consist of inserting the mark directly into the image [6]. They have the advantage of being easy to implement and the insertion and signature detection operations are very fast and have low computation time but are not robust to geometrical attacks [7]. Frequency methods remain more recent methods whose principle is to insert the mark not directly in the image but in the transformed domain. To obtain the marked image, inverse transform is performed [8]. These methods are more resistant to geometric attacks [9]. We propose in this work a watermarking approach for the medical image protection. The integrated watermark contains information about the treated patient as well as data about the 
establishment. This will allow verifying during the extraction that the medical image really corresponds to the treated patient. This approach is applied in the frequency domain. The watermark is integrated into the coefficients obtained by transforms. The wavelet transform of a signal allows representing the signal on a two-dimensional space called the time-scale plane, providing joint information on the signal in time and frequency [10]. The paving of the time-frequency plane induced by this transforms has the particularity of allowing a fine temporal resolution at high frequencies and a fine frequency resolution at low frequencies [11]. This property often allows an interesting analysis of the signal but remains rigid. The interest of this transform is the optimization of the choice of locations and the strength of the signature in the image as well as its multi-scale aspect which offers a more robust distribution to the watermark [12]. Adding the watermark to the lowfrequency coefficients can cause visible perceptual distortion, as the insertion of the watermark in the high-frequency coefficients is vulnerable to some types of attack like compression [13]. To obtain a good compromise between robustness and imperceptibility, the integration is performed in the medium frequencies of the image. To experiment this two approaches, we used the COVID-19 radiography database [14], and the proposed schemes were applied to watermark the radiographic images of this base. Distortion and similarity measurements (between the original and watermarked images) were then calculated to determine the imperceptibility of our approaches. To determine the robustness of our approaches, the most commonly used image watermarking attacks (Compression, filtering...etc.) are applied to the watermarked images. The results obtained will thus allow us to determine the most appropriate approach to the medical image protection. The paper is organized as follows, Sect. 2 will review the various related works that we will use for comparison, and Sect. 3 describes the proposed approach. Section 4 will present the experiments conducted and the results obtained. Finally, the conclusion of our work and the perspectives are proposed in Sect. 5.

\section{Background}

The two main areas of representation used in signal processing are time and frequency. The classical tool used to switch from one domain to the other is the Fourier Transform (TF). The TF allows estimating the frequencies present in a signal, but it does not locate the place where these frequencies appear or disappear, because it acts on the entire signal [15]. To have time-frequency type information, it is necessary to calculate the TF of a small window dragged from one end of the signal to the other.
We then obtain a time-frequency representation. The disadvantage of this technique, besides its low joint time/ frequency resolution, is that the window size is constant. It would be more relevant to adapt the size of the analysis window to the local characteristics of the signal: small window when the signal varies rapidly (high frequencies) and larger window when its variations are slow (low frequencies).Wavelet analysis aims to provide a solution to this problem by decomposing the signal on the basis of elementary signals [16]. Wavelets are obtained by dilating and shifting a basic wavelet.

\subsection{The discrete wavelet transforms}

The discrete wavelet transform is a mathematical formalization that allows to easily constructing orthonormal wavelet bases. The principle consists in decomposing the signal to be analyzed into a series of approximation coefficients and details, each series of approximation being decomposed again into approximation and detail. At each level of this decomposition, the detail coefficients correspond to the wavelet coefficients of the signal at a given scale. The Discrete Wavelet Transform (DWT) decomposes an image into four sub-bands: an LL sub-band of approximation coefficients and three sub-bands of horizontal (HL), vertical (LH) and diagonal (HH) detail coefficients [17]. To obtain the coefficients of each sub-band, we can calculate with the Haar filter as follows:

$L L(x, y)=\frac{\mathrm{p}(\mathrm{x}, \mathrm{y})+\mathrm{p}(\mathrm{x}, \mathrm{y}+1)+\mathrm{p}(\mathrm{x}+1, \mathrm{y})+\mathrm{p}(\mathrm{x}+1, \mathrm{y}+1)}{2}$

$L H(x, y)=\frac{\mathrm{p}(\mathrm{x}, \mathrm{y})+\mathrm{p}(\mathrm{x}, \mathrm{y}+1)-\mathrm{p}(\mathrm{x}+1, \mathrm{y})-\mathrm{p}(\mathrm{x}+1, \mathrm{y}+1)}{2}$

$H L(x, y)=\frac{\mathrm{p}(\mathrm{x}, \mathrm{y})-\mathrm{p}(\mathrm{x}, \mathrm{y}+1)+\mathrm{p}(\mathrm{x}+1, \mathrm{y})-\mathrm{p}(\mathrm{x}+1, \mathrm{y}+1)}{2}$

$H H(x, y)=\frac{\mathrm{p}(\mathrm{x}, \mathrm{y})-\mathrm{p}(\mathrm{x}, \mathrm{y}+1)-\mathrm{p}(\mathrm{x}+1, \mathrm{y})+\mathrm{p}(\mathrm{x}+1, \mathrm{y}+1)}{2}$

The information redundancy contained in this transforms can be generally interesting in the context of signal analysis. Indeed, it is interesting as long as the conditions to be checked by the wavelets are not too constraining. The digital reconstruction of the signal is stable. On the other hand, with the introduction of orthonormal wavelet bases, wavelet theory has become important in the sense that this orthonormal base concept remains a key tool in signal processing; it has speed constraints requiring the minimum amount of possible data. [18]. 


\subsection{Non-subsampled contourlet transform}

The non-subsampled contourlet transform (NSCT) is a translation invariant version of the contourlet transform. The contourlet transform uses a Laplacian pyramid for multi-scale decomposition and banks of directional filters for directional decomposition. To ensure translational invariance, the NSCT is implemented using a non-subsampled pyramid structure and non-sub-sampled directional filter banks [19]. The principle is to successively combine two translation-invariant decomposition stages. A non-subsampled pyramidal structure (NSP), which ensures the multi-scale property and a structure of non-subsampled directional filter banks. Non-Subsampled Directional Filter Banks (NSDFB), which allows decomposition according to different orientations [20]. The result (Fig. 1) is flexible, multi-scale, multi-directional, and translation-invariant image decomposition.

\subsection{The non-subsampled shearlet transform}

A shearlet system can be obtained by anisotropic expansion, translation and division of a finite number of generating functions. These operations are performed by applying a translation operator, an anisotropic expansion operator and a division operator. Unlike wavelet transforms, shearlets only concern $2 \mathrm{D}$ or $3 \mathrm{D}$ signals but are unsuitable for 1D signal [21]. Non-Subsampled shearlet transform (NSST) has been applied to serve as the shiftinvariant and multi-scale expansion of the shearlet transform (Fig. 2), and it has the ability to eliminate the downsamplers and up-samplers. Typically, the implementation of the NSST includes two key steps: the application of
Non-Subsampled pyramid (NSP) filter banks and NonSubsampled shearing (NSS) filter banks [22].

The NSST is a shift-invariant version of the shearlet transform. It differs from the shearlet transform by eliminating of the down-samplers and up-samplers. This transform is a fully shift-invariant, multi-scale and multidirectional transform that combines the non-subsampled Laplacian pyramid transform with different combinations of the shearing filters. The analysis of the non-subsampled Laplacian pyramid can be done through an iterative procedure, where $\mathrm{f}$ is an image, $\mathrm{NSLPj}+1$ is the detail coefficients at scale $\mathrm{j}+1, A h_{j}^{1}$ and $A h_{k}^{0}$ are the low-pass and high-pass filters at scales $\mathrm{j}$ and $\mathrm{k}$, respectively.

$N S L P_{j+1}=\left(A h_{j}^{1} \prod_{k=1}^{j-1} A h_{k}^{0}\right) f$

\subsection{The discreet cosine transform}

The discrete cosine transform (DCT) is widely used in digital signal and image processing [23]. It is a mathematical transformation that transforms a set of data from the spatial to frequency domain and vice versa using the IDCT (Inverse Discrete Cosine Transform). The DCT of an $\mathrm{N} \times \mathrm{N}$ block size for $\mathrm{i}, \mathrm{j}=1,2 \ldots \mathrm{N}$ is calculated as follows:

$$
\begin{aligned}
F_{u, v}= & \frac{1}{\sqrt{2 N}} C_{u} \cdot C_{v} \sum_{x=1}^{N} \sum_{y=1}^{N} f_{x, y} \\
& \cdot \cos \left[\frac{(2 x+1) u \pi}{2 N}\right] \cos \left[\frac{(2 y+1) v \pi}{2 N}\right]
\end{aligned}
$$

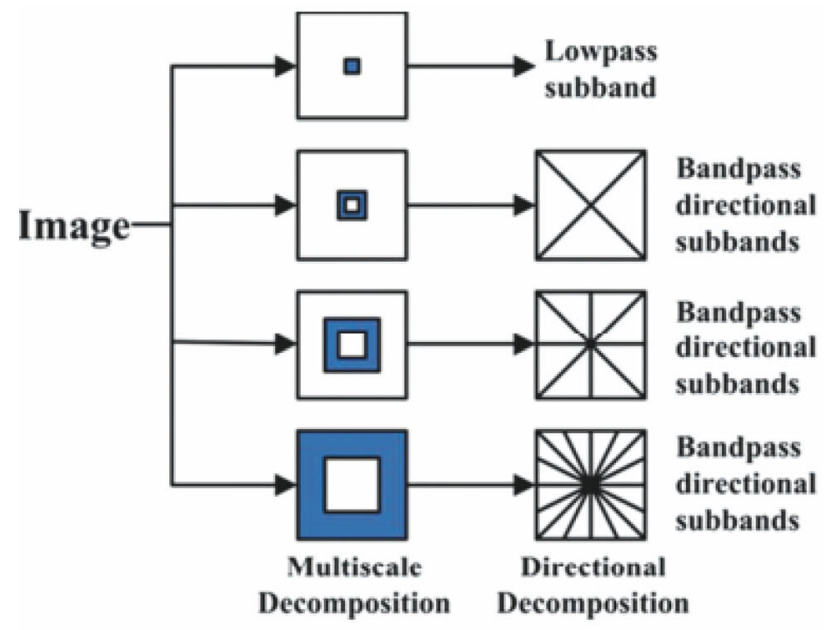

(a)

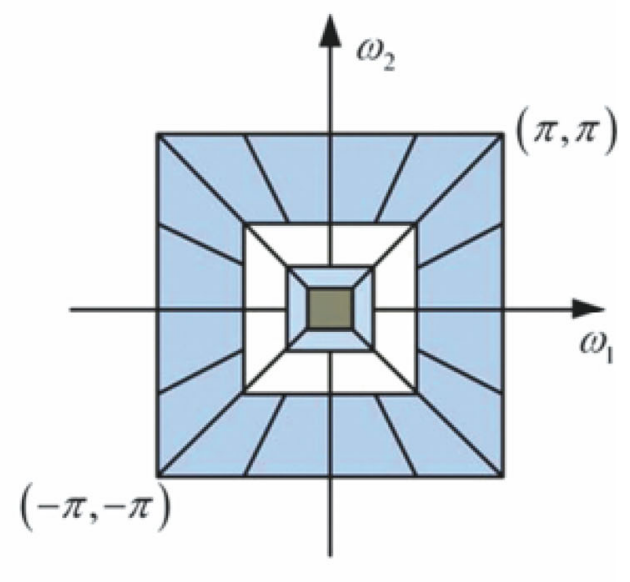

(b)

Fig. 1 Non-subsampled contourlet transform: a NSFB structure, $\mathbf{b}$ idealized frequency partitioning 
Fig. 2 Non-subsampled shearlet transform

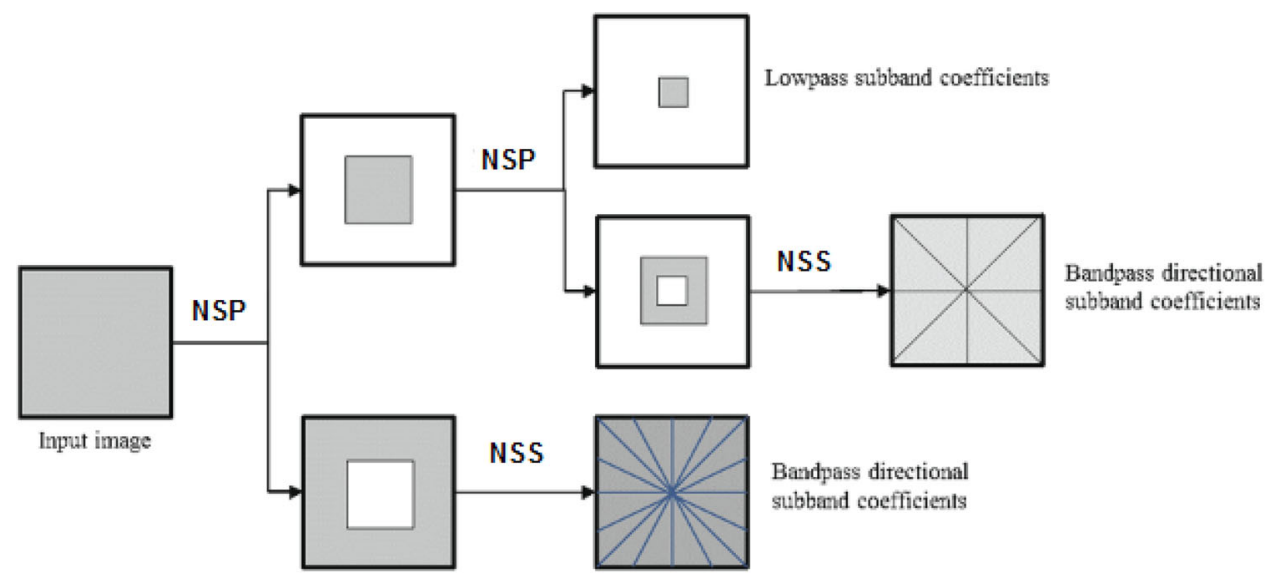

$\mathrm{Cu}=\left\{\begin{array}{cc}\frac{1}{\sqrt{2 N}} & \text { if } u=0 \\ 1 & \text { if } u>0\end{array}\right.$

Next, thresholding is performed to increase the efficiency of the compression. If the absolute values of the (non-zero) coefficients of the DCT matrix obtained are below a certain threshold [24], these coefficients will be eliminated (set to zero). The quantization phase then reduces the importance of all DCT coefficients by dividing them by a quantization step and replacing them with their quotient with this step. Quantification is therefore a simple Euclidean division of the DCT coefficients by a certain divisor (the step), which replaces the original coefficients by the quotient of the division. It is therefore at this step that we will lose part of the information, because after quantization the rest of the division is lost [25]. To calculates the quantization matrix, the quantization value $Q$ is chosen ( $Q$ represents the number of bits needed to code each element of the DCT matrix). The two MAX and MIN values of the DCT matrix (DCTmax, DCTmin) are determined. Next, the DCTQ matrix is calculated by the following formula:

$\operatorname{DCT} Q(i, j)=\frac{\left(-1+2^{\mathrm{Q}}\right)(\mathrm{DCT}(\mathrm{i}, \mathrm{j})-\mathrm{DCTmin})}{\mathrm{DCTmax}-\mathrm{DCTmin}}$

\subsection{Schur decomposition}

In linear algebra, Schur decomposition [26] of a complex square matrix $\mathrm{M}$ is a decomposition of the form:

$M=U \times S \times U^{\prime}$,

where $U$ is a unitary matrix $\left(U \times U^{\prime}=I\right)$ and $S$ the upper triangular matrix.

The successful application of singular values decomposition in digital watermarking suggests the use of Schur decomposition for the same purpose. However, Schur decomposition represents an alternative to the singular values decomposition. Indeed, singular value decomposition requires about $11 \mathrm{~N}^{3}$ flops as compared to a Schur decomposition which requires only $8 \mathrm{~N}^{3} / 3$. This represents about three times less computing time. However, the calculations should not be expected to be reduced by the same amount. This is mainly due to the fact that the integration and extraction procedures involve operations other than the Schur decomposition.

\subsection{Related works}

\subsubsection{Watermarking approach using NSST}

Wang et al. presents a novel robust watermarking algorithm in Non-Subsampled Shearlet Transform (NSST) domain using quaternion polar harmonic transform (PHT) and least squares support vector regression (LS-SVR). In this approach, the low pass subband of $\mathrm{Y}, \mathrm{U}$, and $\mathrm{V}$ color components are used to embed watermark information in NSST domain [27]. To more significantly improve the resilience of the watermarking algorithm against image processing attacks, the author adopt the redundant watermark embedding strategy, in which the watermark information is embedded repeatedly into the non-overlapping low frequency NSST coefficients blocks. Zheng et al. propose an adaptive embedding strength watermarking algorithm based on shearlets' capture directional features [28]. In this approach, a DWT is first applied to obtain the subband decomposition. Then, a NSST is applied to the low frequency sub-band. Singular value decomposition (SVD) is applied to the watermark image, and then the principle components are extracted. The principle components, which represent the unique features of the image, are embedded into the host image to eliminate the false positive problem. Favorskaya \&al propose a robust watermarking scheme based on the prerequisites of the HVS. In this approach, a perceptual channel decomposition of the 
host image is done to provide the appropriate regions for embedding. A non-subsampled shearlet transform is then applied to the selected regions [29]. SVD is then applied to the obtained NSST coefficient. Finally, the watermark bits are embedded by replacing the least significant bit of the singular values. To enhance the security, the watermark is scrambled by the Arnold's transform.

\subsubsection{Watermarking approach using NSCT}

Chen et al. present a robust blind watermarking scheme based on Non-Subsampled Contourlet Transform and Singular Value Decomposition. In this approach, the original image is decomposed using the NSCT to obtain the coarse sub-band and band-pass sub-bands [30]. The resulting low pass approximation sub-band is decomposed into bloc $(8 \times 8)$ without overlapping. Singular value decomposition is then applied to each bloc. Each singular value is used to embed one bit watermark information. Gao et al. propose a non-subsampled Contourlet Transform based watermarking scheme [31]. In this approach, the cover image is transformed by NSCT to obtain low-pass subband. For enhancing the algorithm robustness, the directional subband with maximum energy is selected in mid-frequency subbands. For the watermark embedding, the region with the most abundant texture is selected based on the characteristics of human visual system (HVS). To enhance the watermark robustness, watermark bit information is repeatedly embedded into coefficients of a block selected randomly by logistic chaotic map with a key.

\subsubsection{Watermarking approach using DCT}

$\mathrm{Hu}$ et al. proposes a blind watermarking scheme based on discrete cosine transform (DCT) to integrate binary information into images [32]. This scheme modulates the Partially Signed Average (PSA) of some DCT coefficients derived from an $8 \times 8$ picture block. The variation margin of each coefficient is regulated by consulting a JPEG quantization table to reinforce the imperceptibility of the watermark. In the approach proposed by Moosazadeh et al. a DCT of the image is calculated [33], then, the parameters and position of the coefficients to be modified is determined automatically by a method of Teaching-LearningBased Optimization. The proposed method uses TLBO, which has rarely been applied in watermarking and allows to automatically determining the integration parameters and the appropriate position for the watermark insertion. Assini et al. [34] proposes a robust watermarking scheme combining discrete wavelet transform (DWT), discrete cosine transform (DCT) and singular value decomposition (SVD). A DWT is applied to the medical image to obtain different frequency sub-bands. Then a
DCT is applied to the HH sub-band. An SVD is then applied to the obtained DCT coefficients. Finally, the watermark is integrated into the singular values obtained from the $\mathrm{HH}$ high-frequency sub-band of the medical image.

\subsubsection{Watermarking approach using DWT}

Verma et al. present a hybrid technique [35] combining DWT and SVD for the watermarking of medical images (to integrate the advantages of both techniques). A DWT is applied to the image to obtain the different frequency subbands. Singular value decomposition is then applied to the LL sub-band. The watermark is integrated into the S-value using a scaling factor. The combination of the modified part $\mathrm{S}$ and the original parts $\mathrm{U}$ and $\mathrm{V}$ provides a modified LL sub-band. An IDWT with the modified LL sub-band is finally applied to obtain the watermarked image. Anand et al. proposes a multi-watermark embedding method for medical image [36]. The embedding watermark is composed of a text and an image. A four sub-band decomposition of the host image is first performed by applying a DWT. An SVD is applied to the HH sub-band, and the text is embedded in the singular value $S$. The image watermark is divided into two sub images, these two parts are then integrated (after applying an SVD to both sub bands) into the singular $\mathrm{S}$ values of the HL and LH sub bands of the cover image. To enhance security, the watermarked image is then encrypted using Hyperchaotic encryption algorithm. In the approach presented by Gangadhar et al. [37], a subband decomposition of the medical image is first performed by applying a DWT. Then, an SVD is applied to the LL subbands. To determine the most suitable regions for the integration process, the particle swarm optimization algorithm is then applied to the LL subbands. Alshanbari et al. [38] combine two different watermarking insertion methodologies. In this work, multiple watermarks are integrated into the medical image. A secret key is first generated based on SHA-256 algorithm using the image ROI. The ROI is compressed with Lempel-Ziv-Welch (LZW) algorithm and then, combined with signature information. The generated watermark is then inserted into the RONI region of host image. Devi et al. [39] present a blind watermarking scheme that combines DWT and SVD. A DWT is applied to the blue component of the cover image; the resulting LL sub-band is then decomposed into $8 \times 8$ blocks. For each block, a fast Walsh Hadamard transformation is applied. Singular value decomposition is then applied to the blocks of the LL sub-band formed by the Hadamard transform. The watermark bits are then integrated into the singular values obtained. To increase security, the watermark image is scrambled using the Arnold Transform. 


\section{Proposed watermarking scheme}

A watermarking scheme generally consists of three important processes, the mark generation, the integration process and the extraction process [40].

\subsection{Watermark generation}

The watermark used in our approach consists of patient information and image acquisition data. This information is concatenated and a hash is computed using the MD5 algorithm (Fig. 3). This hash will guarantee the integrity of the extracted watermark. The extraction of this data will precisely allow identifying the patient and avoiding any confusion.

\subsection{DWT-Schur watermark integration and extraction process}

For the watermark integration, a DWT is first applied to the image to obtain the four subbands LL, HL, LH and HH. This representation makes possible to distinguish very specific regions around the image contours and textured areas. These regions correspond to areas where there is a high density of significant (or dominant) wavelet coefficients. Some works are based on modifying the coefficients of all the sub-bands; others rely on inserting in HH or LL. To improve robustness to some attacks like compression, filtering and scaling while maintaining a good imperceptibility rate. In our approach the $\mathrm{LH}$ and $\mathrm{HL}$ subbands are used for the watermark integration (Fig. 4). Schur decomposition is then applied to the LH and HL subbands. The upper triangular matrix is then used for the watermark bit integration. Every three successive values of this matrix are modulated to integrate the watermark bits according to the rules in Fig. 3. These rules allow modifying only the parity of a single value, so for the integration of two bits of the watermark a single bit will be modified, the inequality must be modified to match the equations to the watermark bits, this will allow the extraction of the watermark in blind mode (without using the original image).

The receiver will just have to apply equations (Fig. 5) to get the watermark bits without using the original image. Finally, an inverse Schur decomposition is applied to obtain the modified subbands. HL and LH, an inverse DWT is then applied to the four subbands LL, HL, LH and $\mathrm{HH}$ to obtain the watermarked image.

For the extraction process, a DWT is applied to the watermarked image, as shown in the diagram block in
Fig. 6. Then, Schur decomposition is applied to the resulting LH and HL subbands. As for the DCT-Schur algorithm, the watermark bits are extracted from the upper triangular matrix.

\subsection{DCT-Schur watermark integration and extraction process}

Calculating the DCT over the entire image would take a considerable time. To avoid this, in our approach, we apply DCT on fixed-length blocks $(8 \times 8$ pixels $)$, this choice gives a better compromise between quality and computation time. The image is thus divided into blocks $(8 \times 8)$ without overlapping (Fig. 7), and then a 2D DCT is applied to each block. This transformation will allow separating the low frequencies from the high frequencies. All the information of the image is in the low frequencies. On the other hand, the details of the image are located in the high frequencies. If the mark is inserted in the low frequencies, the watermark will be robust but the mark will be visible. If the mark is inserted in the high frequencies, it will be imperceptible but the watermark will be less robust because of the compression operations. To find a compromise between imperceptibility and robustness, medium frequencies are selected for the integration process. Schur decomposition is then applied to the medium frequencies obtained; the upper triangular matrix is then used for the watermark bits integration. Every three successive values of this matrix are modulated to integrate the watermark bits according to the rules in Fig. 5. Finally, an inverse Schur decomposition is applied to obtain the modified mid band frequency coefficients. These blocks are combined with the other blocks, and then an Inverse DCT is applied to obtain the watermarked image.

For the extraction process, a DCT is applied to the watermarked image, as shown in the diagram block in Fig. 8. Then, Schur decomposition is applied to the mid band frequency. The watermark bits are then extracted from the upper triangular matrix.

\subsection{NSCT-Schur watermark integration and extraction process}

For watermark integration, a non-subsampled contourlet transform is applied to the medical image. The NSCT allows obtaining the approximate low-pass subband $\mathrm{B}$ and directional subbands $\mathrm{C}$. The watermark bits are then integrated into the directional subbands coefficients with maximum energy in the mid-frequency subbands. The
Fig. 3 Watermark generation process

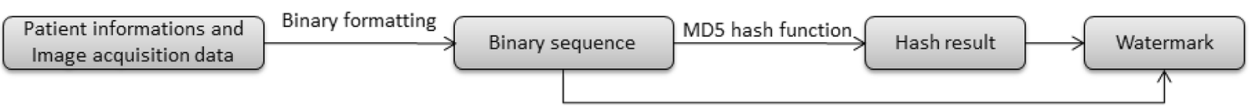


Fig. 4 Integration process for the DWT-Schur algorithm

Fig. 5 Substitution rules

Fig. 6 Extraction process for the DWT-Schur algorithm

Fig. 7 Integration process for the DCT-Schur algorithm

Fig. 8 Extraction process for the DCT-Schur algorithm
Apply Schur decomposition

on LH and HL Sub-bands

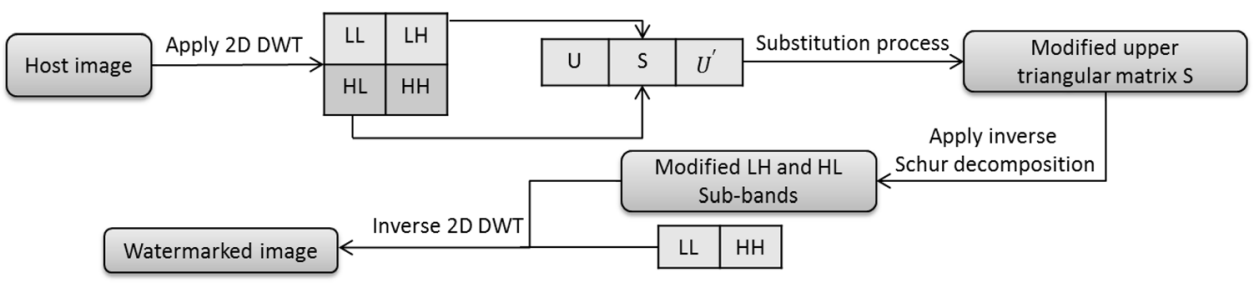

Three succes sive coefficients $\mathrm{S} 1, \mathrm{~S} 2$ and $\mathrm{S} 3$

Two watermatk bits $\mathrm{X}$ and $\mathrm{Y}$

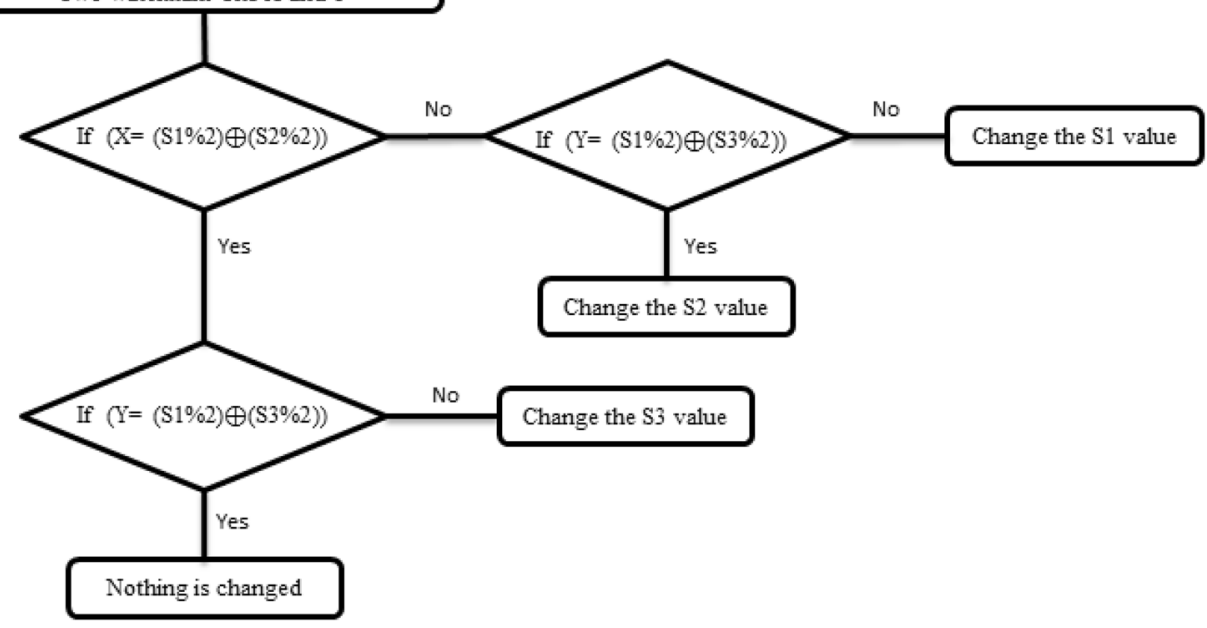

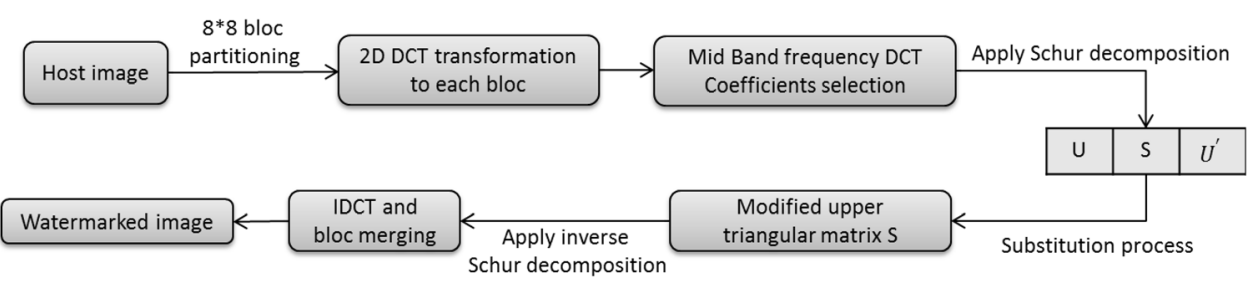

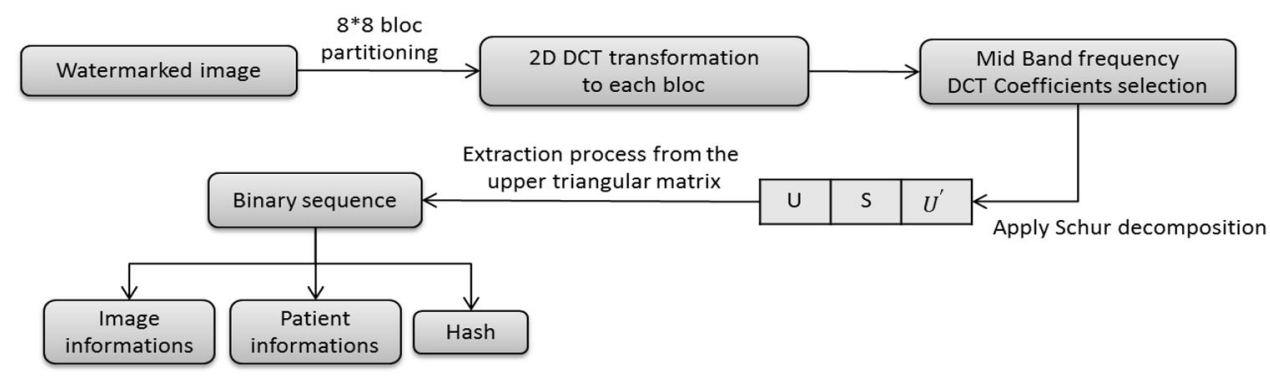


calculation of the subband energies is calculated according to the formula:

$\mathrm{E}=\frac{1}{\mathrm{M} \times \mathrm{N}} \sum_{m=1}^{M} \sum_{n=1}^{N}(C(m, n))^{2}$,

where $\mathrm{M}$ and $\mathrm{N}$ represent respectively the width and height of the directional subband.

Schur decomposition is then applied to the obtained medium frequencies (Fig. 9), the upper triangular matrix is then used for the watermark bits integration. Every three successive values of this matrix are modulated to integrate the watermark bits according to the rules in Fig. 10.

Finally, an inverse Schur decomposition is applied to obtain the modified mid band frequency coefficients. These blocks are combined with the other blocks, and then an Inverse NSCT is applied to obtain the watermarked image. For the extraction process, a NSCT is applied to the watermarked image, as shown in the diagram block in Fig. 11. Then, a Schur decomposition is applied to the mid band frequency. The watermark bits are then extracted from the upper triangular matrix. The binary sequence obtained is divided into three parts, and the first part represents the patient's personal information, and the second part contains the identity of the doctor in charge of this patient as well as the image acquisition parameters. The third part contains the hash of the two first parts; this binary sequence is very useful to verify the integrity of the extracted data. This ensures the patient or image information has not been altered during transfer or by an unauthorized entity.

\subsection{NSST-Schur watermark integration and extraction process}

For the watermark integration, the image is divided into blocks $(8 \times 8)$ without overlapping, and then a NSST is first applied to the image. This representation makes possible to distinguish very specific regions around the image contours and textured areas. These regions correspond to areas where there is a high density of significant (or dominant) coefficients.

Schur decomposition is then applied to the low frequency NSST sub-bands (Fig. 12). The upper triangular matrix is then used for the watermark bit integration. Every three successive values $(\mathrm{C} 1, \mathrm{C} 2$ and $\mathrm{C} 3)$ of this matrix are modulated to integrate the watermark bits $\mathrm{X}$ and $\mathrm{Y}$ according to this rule:

$\left\{\begin{array}{l}C 1++, \text { If }((X \neq(C 1-C 2) \% 2) \text { and }(Y \neq(C 1-C 3) \% 2)) \\ C 2++, \text { If }((X=(C 1-C 2) \% 2) \text { and }(Y \neq(C 1-C 3) \% 2)) \\ C 3++, \text { If }((X \neq(C 1-C 2) \% 2) \text { and }(Y=(C 1-C 3) \% 2))\end{array}\right.$

These rules allow modifying only the parity of a single value, so for the integration of two bits of the watermark a single bit will be modified, the inequality must be modified to match the equations to the watermark bits, this will allow the extraction of the watermark in blind mode (without using the original image). Finally, an inverse Schur decomposition is applied to obtain the modified Low frequency subbands. These blocks are combined with the other blocks, and then an Inverse NSST is applied to obtain the watermarked image. For the extraction process, a NSST is applied to the watermarked image, as shown in the diagram block in Fig. 13. Next, Schur decomposition is applied to the Low frequency subbands. The watermark bits are then extracted from the upper triangular matrix.

\section{Experimental results and discussions}

In this section, several experiments are taken to evaluate the performance of our proposed watermarking scheme. The experiments were performed using images from the COVID-19 radiography database (Fig. 14). This database contains chest X-ray images for COVID-19 positive cases along with Normal and Viral Pneumonia images.

In this release, there are 219 COVID-19 positive images, 1341 normal images and 1345 viral pneumonia images. All the images are in Portable Network Graphics (PNG) file format and resolution is 1024-by-1024 pixels. In this experiment, all methods are performed on the same platform of 2.27GHZ CPU, 2.00 GB RAM, Win 7 and MATLAB 8.5.0 (R2015a). The experimental results are divided into two parts: the first is focused on measuring the imperceptibility while the second is dedicated to the robustness analysis against some types of attacks. In medical images, the imperceptibility concept remains the most important property and differs from the case of general public multimedia.

Indeed, watermarking can hide subtle signs or introduce new artifacts that will falsify the image's interpretation.

Fig. 9 Integration process for the NSCT-Schur algorithm

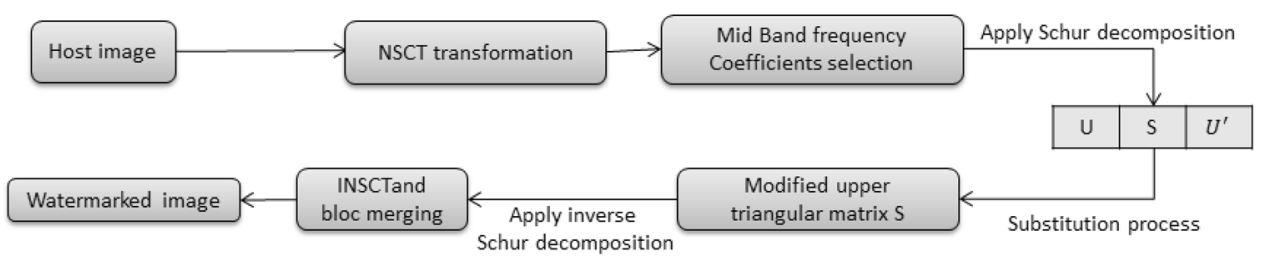


Fig. 10 Substitution rules

Fig. 11 Extraction process for the NSCT-Schur algorithm

Fig. 12 Integration process for the NSCT-Schur algorithm

Fig. 13 Extraction process for the NSCT-Schur algorithm
Three successive coefficients $\mathrm{C} 1, \mathrm{C} 2$ and

C3. Two watermark bits $\mathrm{X}$ and $\mathrm{Y}$
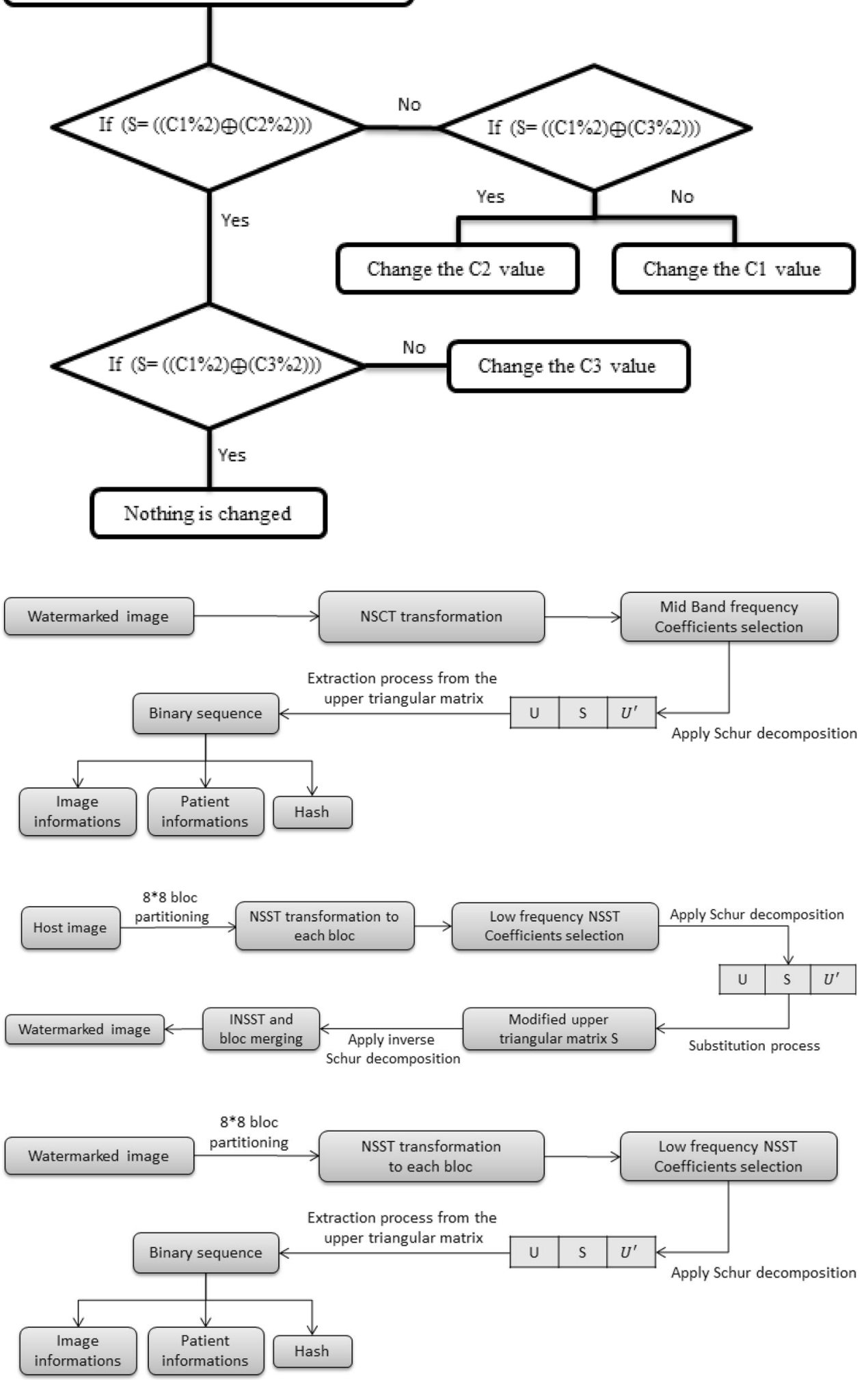
Fig. 14 Medical images used for the experimentations
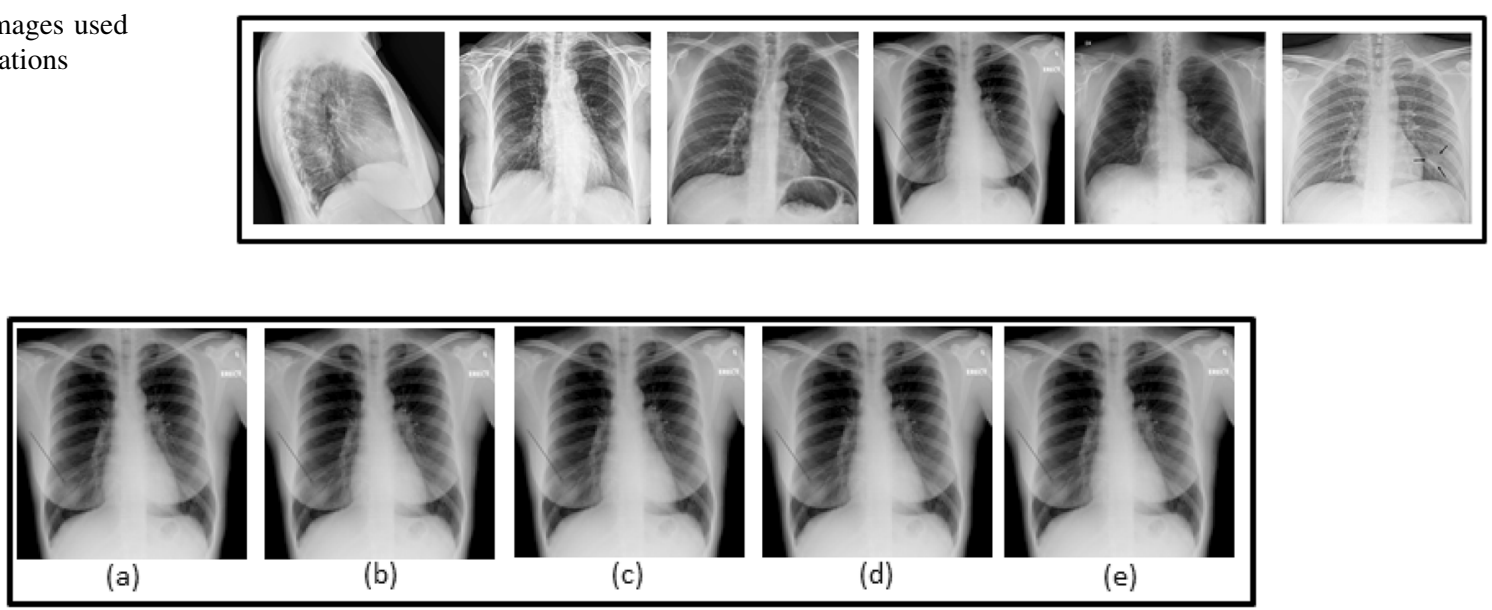

Fig. 15 Watermarking results, a original image, b image watermarked using DCT-Schur, c image watermarked using DWT-Schur, d Image watermarked using NSST-Schur, e image watermarked using NSCT-Schur

The consequences will be more or less serious, even lifethreatening. As we can see on Fig. 15, it is difficult to make the difference between the original and watermarked images; the degradation of the watermarked images is visually imperceptible.

\subsection{Imperceptibility test}

To evaluate the imperceptibility of our approaches, we used the PSNR (peak signal to noise ratio) which is a distortion measure widely used in digital imaging and especially in image compression [41]. The aim is to quantify the performance of encoders by measuring the quality of the watermarked image compared to the original image. While the PSNR is useful to measure the proximity of the watermarked image to the original, it does not take into account the visual quality of reconstruction and cannot be considered as an objective measure of the visual quality of an image [42]. For this purpose the structural similarity index measurement (SSIM) is calculated. Also, after extraction of the mark, the normalized correlation coefficient (NCC) is calculated using the original mark and the extracted mark. This coefficient is used to evaluate the existence and accuracy of the extracted mark. The number of values changed (NVC) by the integration process is also calculated between the original and watermarked image. The standard deviation (STD) is also calculated. The average values obtained for the 200 images used will thus be represented in all the following tables.

As we can see in Table 1, the PSNR rate offered by the DWT-Schur approach is relatively higher than that of the DCT-Schur algorithm, as well as the mean square error which is slightly lower for the DWT-Schur approach, the rate of structural similarity index measurement is almost identical, however the number of values modified by the
Table 1 Imperceptibility results

\begin{tabular}{llllll}
\hline & PSNR & SSIM & NVC $(\%)$ & STD & NCC \\
\hline NSST-Schur & 42.85 & 0.9998 & 42.18 & 0.704 & 0.9923 \\
NSCT-Schur & 44.98 & 0.9998 & 48.97 & 0.131 & 0.9947 \\
DWT-Schur & 49.20 & 0.9998 & 54.82 & 0.024 & 0.9995 \\
DCT-Schur & 47.98 & 0.9997 & 45.48 & 0.329 & 0.9989 \\
\hline
\end{tabular}

DCT-Schur approach is significantly lower than the rate of values modified by the DWT-Schur algorithm. To evaluate our two proposed algorithms, the imperceptibility results obtained are compared with the approaches presented in Sect. 2 (Table 2). However, since the watermark used is composed of a hash, the alteration of a single bit of this hash will result in the loss of the embedded fingerprint message. This will then make impossible the verification of the extracted watermark's integrity.

\subsection{Robustness test}

In order to preserve the patient's or doctor's data, the watermarking algorithm must resist to the various attacks. In order to verify the robustness of the proposed algorithms, we tested both algorithms against various types of attacks, including JPEG compression, Gaussian noise, Sal and Pepper noise, median filter and other attacks. The normalized cross-correlation is then calculated between the original and extracted watermark (Table 3 ).

\subsection{Discussion}

The image decomposition into sub-bands has allowed separating the low-frequency components, which constitute 
Table 2 Imperceptibility results comparison with related work

\begin{tabular}{|c|c|c|c|c|c|c|c|}
\hline & \multicolumn{3}{|c|}{ DCT-based approach } & \multicolumn{4}{|c|}{ DWT-based approach } \\
\hline & Hu et al. & Moosazadeh et al. & DCT-Schur & Anand et al. & Gangadhar et al. & Devi et al. & DWT-Schur \\
\hline \multirow[t]{3}{*}{ PSNR } & 40.48 & 40.76 & 47.98 & 44.19 & 43.52 & 54.64 & 49.20 \\
\hline & \multicolumn{3}{|c|}{ NSCT-based approach } & \multicolumn{4}{|c|}{ NSST-based approach } \\
\hline & Gao et al. & Chen et al. & NSCT-Schur & Favorskaya et al. & Zheng et al. & Wang et al. & NSST-Schur \\
\hline PSNR & 42.47 & 47.30 & 44.98 & 39.63 & 38.01 & 38.52 & 42.85 \\
\hline
\end{tabular}

Table 3 Robustness comparison with related work

\begin{tabular}{|c|c|c|c|c|c|c|c|c|c|}
\hline & \multicolumn{3}{|c|}{ DWT-based approach } & \multicolumn{2}{|c|}{ DCT-based approach } & \multicolumn{2}{|c|}{ NSST-based approach } & \multicolumn{2}{|c|}{ NSCT-based approach } \\
\hline & $\begin{array}{l}\text { Devi } \\
\text { et al. }\end{array}$ & $\begin{array}{l}\text { Anand } \\
\text { set al. }\end{array}$ & $\begin{array}{l}\text { DWT- } \\
\text { Schur }\end{array}$ & $\begin{array}{l}\text { Moosazadeh } \\
\text { et al. }\end{array}$ & $\begin{array}{l}\text { DCT- } \\
\text { Schur }\end{array}$ & $\begin{array}{l}\text { Zheng } \\
\text { et al. }\end{array}$ & $\begin{array}{l}\text { NSST- } \\
\text { Schur }\end{array}$ & $\begin{array}{l}\text { Chen } \\
\text { et al. }\end{array}$ & $\begin{array}{l}\text { NSCT- } \\
\text { Schur }\end{array}$ \\
\hline $\begin{array}{l}\text { Histogram } \\
\text { equalization }\end{array}$ & 0.8764 & 0.7223 & 0.8555 & - & 0.8607 & 0.9940 & 0.9274 & - & 0.8607 \\
\hline Gaussian noise & 0.9953 & 0.9803 & 0.9733 & - & 0.9412 & 0.9930 & 0.9922 & 0.8438 & 0.9914 \\
\hline Sharpening & 0.9253 & 0.6506 & 0.7173 & - & 0.7693 & 0.9923 & 0.8784 & - & 0.7644 \\
\hline Average filtering & 0.769 & 0.986 & 0.9567 & 0.8866 & 0.8854 & 0.9969 & 0.9574 & 0.8965 & 0.9179 \\
\hline Cropping & 0.8141 & 0.3586 & 0.5829 & 0.8219 & 0.6615 & 0.9967 & 0.8541 & 0.7363 & 0.7115 \\
\hline JPEG compression & 0.9965 & 0.9388 & 0.9617 & - & 0.9854 & 0.9968 & 0.9699 & - & 0.9430 \\
\hline $\begin{array}{l}\text { Salt and pepper } \\
\text { noise }\end{array}$ & - & 0.9251 & 0.8936 & 0.8672 & 0.9141 & 0.9848 & 0.9294 & 0.7539 & 0.8741 \\
\hline Scaling & - & 0.7157 & 0.7475 & 0.9489 & 0.7793 & 0.9712 & 0.8917 & 0.9551 & 0.8122 \\
\hline
\end{tabular}

an insertion space for the signature less sensitive to modifications. On the other hand, the spatial content of the image remains preserved after a multi-resolution transformation, this similar content can then be used to locate the mark after a geometric transformation. In this work, we also reviewed recent transformations. These multi-scale decompositions, operating according to a multitude of frequency orientations, provide a satisfactory compromise between the scattered (or compact) representation of visual information and the perceptual quality of the reconstructed image. In particular, the Non-Subsampled Contourlet Transform. The NSCT represent a discrete version, therefore adapted to digital images, which offers better directional selectivity and maintains the property of translational invariance. Unlike the contourlet transform, NSCT is a fully translation-invariant, multi-scale, multi-directional image decomposition that has better frequency selectivity and fast implementation. However, it is important to highlight that the wavelet transform is not optimal for modeling the anisotropic features of an image. This is due to the isotropic nature of the operators used. Since these operators process the two axes of the image equally, 2D high-frequency wavelets are well unsuited for singularities other than vertical, horizontal or diagonal singularities. The shearlet transform, which is a recent decomposition based on anisotropic operators, has been used to overcome this limitation. The imperceptibility results obtained remain acceptable. The use of the DCT in the first approach allows for high coefficient dissociation, as most of the information contained in an image is concentrated in a reduced number of coefficients, which facilitates integration. In addition, the signal energy included in the transformed domain will be distributed over all pixels in the spatial domain, which considerably improves the imperceptibility. Representations at different resolutions in the second approach (DWT-Schur) allow the extraction of the image's features into a small number of coefficients, while precisely locating the discontinuities. Hence, the most significant areas of the image are highlighted. The robustness results obtained are practically similar for the four approaches. Both approaches allow generating a watermark robust to filtering and Gaussian noise attacks; however we can see that the DCT approach is more robust to compression attacks. The discrete cosine transform makes the watermark more robust to JPEG compression, since they exploit the same space used for image coding. It also reduces the spatial 
correlation between pixels in an image. In addition, each block is independently transformed in the DCT domain which improves the algorithm's robustness. Moreover, Eigen values in the Schur decomposition are remarkably stable, so the use of the upper triangular matrix for the watermark integration allows a better robustness.

\section{Conclusion}

The digital image watermarking finds an application in the medical imaging domain and particular in the field of telemedicine. Indeed, given the importance and growth of the practice of remote medicine, watermarking can be proposed to contribute to the security of medical images shared on the Internet. The medical image, given its specificities, must be handled with considerable precautions. An image degradation which would seem insignificant is often not accepted in this field because it could lead to an incorrect diagnosis. Currently, a great interest is given to the substitutive watermark which represents the methods for which the mark is not added but substituted and formed by modification of certain image components. In this work we have proposed a substitutive watermarking approach for the medical image protection. These schemes allow the protection of the patient's information and therefore ensure the confidentiality of personal data. Moreover, these schemes ensure the integrity of the hidden data through a hash embedded in the image. Imperceptibility and robustness experimental results show that the proposed methods maintain a high quality of watermarked images and are very robust against several conventional attacks. However, the principal disadvantage of this approach is that if the hash is altered, it makes impossible to verify the integrity of the extracted information. The proposed scheme can be integrated into a medical document authentication application where any user can check whether a document from the database is authentic or not.

\section{Compliance with ethical standards}

Conflict of interest The authors declare that they have no known competing financial interests or personal relationships that could have appeared to influence the work reported in this paper.

Ethical approval This article does not contain any studies with human participants or animals performed by any of the authors.

\section{References}

1. Tamilarasi, K., Jawahar, A.: Medical data security for healthcare applications using hybrid lightweight encryption and swarm optimization algorithm. Wirel. Pers. Commun. 114(3), 1865-1886 (2020). https://doi.org/10.1007/s11277-020-07229-x

2. Balasamy, K., Ramakrishnan, S.: An intelligent reversible watermarking system for authenticating medical images using wavelet and PSO. Clust. Comput. 22(2), 4431-4442 (2019). https://doi.org/10.1007/s10586-018-1991-8

3. Boussif, M., Aloui, N., Cherif, A.: DICOM imaging watermarking for hiding medical reports. Med. Biol. Eng. Comput. 58(11), 2905-2918 (2020). https://doi.org/10.1007/s11517-02002269-8

4. Kahlessenane, F., Khaldi, A., Kafi, R., Euschi, S.: A DWT based watermarking approach for medical image protection. J. Ambient Intell. Hum. Comput. (2020). https://doi.org/10.1007/s12652020-02450-9

5. Balu, S., Nelson Kennedy Babu, C., Amudha, K.: Secure and efficient data transmission by video steganography in medical imaging system. Clust. Comput. 22(2), 4057-4063 (2019). https://doi.org/10.1007/s10586-018-2639-4

6. Khaldi, A.: Diffie-Hellman key exchange through steganographied images. Law State Telecommun. Rev. 10(1), 147-160 (2018). https://doi.org/10.26512/lstr.v10i1.21504

7. Gul, E., Ozturk, S.: A novel triple recovery information embedding approach for self-embedded digital image watermarking. Multimedia Tools Appl. 79(41), 31239-31264 (2020). https://doi. org/10.1007/s11042-020-09548-4

8. Senthilnathan, T., Prabu, P., Sivakumar, R., Sakthivel, S.: An enhancing reversible data hiding for secured data using shuffle block key encryption and histogram bit shifting in cloud environment. Clust. Comput. 22, 12839-12847 (2019). https://doi. org/10.1007/s10586-018-1765-3

9. Khaldi, A.: Steganographic techniques classification according to image format. Int. Ann. Sci. 8(1), 143-149 (2020). https://doi.org/ 10.21467/ias.8.1.143-149

10. Devanna, H., Satish Kumar, G.A.E., Giri Prasad, M.N.: A spatiofrequency orientational energy based medical image fusion using non-sub sampled contourlet transform». Clust. Comput. 22, 11193-11205 (2019). https://doi.org/10.1007/s10586-017-1351-0

11. Hu, H.-T., Chang, J.-R.: Efficient and robust frame-synchronized blind audio watermarking by featuring multilevel DWT and DCT. Clust. Comput. 20(1), 805-816 (2017). https://doi.org/10. 1007/s10586-017-0770-2

12. Fares, K., Amine, K., Salah, E.: A Robust blind color image watermarking based on Fourier transform domain. Optik 208, 164562 (2020). https://doi.org/10.1016/j.ijleo.2020.164562

13. Kavitha, C., Sakthivel, S.: An effective mechanism for medical images authentication using quick response code. Clust. Comput. 22(2), 4375-4382 (2019). https://doi.org/10.1007/s10586-0181905-9

14. Rayachoti, E., Tirumalasetty, S., Prathipati, S.C.: SLT based watermarking system for secure telemedicine. Clust. Comput. 23, 3175-3184 (2020). https://doi.org/10.1007/s10586-020-03078-2

15. Chowdhury, M.E.H., Rahman, T., Khandakar, A., Mazhar, R., Kadir, M.A., Mahbub, Z.B., Islam, K.R.: Can AI help in screening viral and COVID-19 pneumonia? IEEE Access 8, 132665-132676 (2020). https://doi.org/10.1109/ACCESS.2020. 3010287

16. Rajkumar, R., Vasuki, A.: Reversible and robust image watermarking based on histogram shifting. Clust. Comput. 23, 12313-12323 (2019). https://doi.org/10.1007/s10586-017-1614-9

17. Salah, E., Amine, K., Redouane, K., Fares, K.: A Fourier transform based audio watermarking algorithm. Appl. Acoust. 172, 107652 (2021). https://doi.org/10.1016/j.apacoust.2020.107652

18. Balasamy, K., Suganyadevi, S.: A Fuzzy based ROI selection for encryption and watermarking in medical image using DWT and SVD. Multimedia Tools Appl. (2020). https://doi.org/10.1007/ s11042-020-09981-5 
19. Giri, K.J., Quadri, S.M.K., Bashir, R., Bhat, J.I.: DWT based color image watermarking: a review. Multimedia Tools Appl. 79(43), 32881-328895 (2020). https://doi.org/10.1007/s11042020-09716-6

20. Saha, C., Foisal Hossain, Md., Asadur Rahman, Md.: NSCTbased robust image watermarking in DC components of APDCBT using singular value decomposition. Iran J. Comput. Sci. (2020). https://doi.org/10.1007/s42044-020-00070-2

21. Zhong, Z., Gao, W., Khattak, A.M., Wang, M.: A novel multisource image fusion method for pig-body multi-feature detection in NSCT domain. Multimedia Tools Appl. 79(35), 26225-26244 (2020). https://doi.org/10.1007/s11042-020-09044-9

22. Niu, P., Shen, X., Song, Y., Liu, Y., Wang, X.: Locally optimum watermark decoder in NSST domain using RSS-based Cauchy distribution. Multimedia Tools Appl. 79(43), 33071-33101 (2020). https://doi.org/10.1007/s11042-020-09621-y

23. Zhang, Z., Xi, X., Luo, X., Jiang, Y., Dong, J., Xiaojun, Wu.: Multimodal image fusion based on global-regional-local rule in NSST domain. Multimedia Tools Appl. (2020). https://doi.org/ 10.1007/s11042-020-09647-2

24. Abdulrahman, A.K., Ozturk, S.: A novel Hybrid DCT and DWT based robust watermarking algorithm for color images. Multimedia Tools Appl. 78(12), 17027-17049 (2019). https://doi.org/ 10.1007/s11042-018-7085-Z

25. Kanhe, A., Gnanasekaran, A.: A blind audio watermarking scheme employing DCT-HT-SD technique. Circuits Syst. Signal Process. 38(8), 3697-3714 (2019). https://doi.org/10.1007/ s00034-018-0994-2

26. Su, Q., Zhang, X., Wang, G.: An improved watermarking algorithm for color image using Schur decomposition. Soft Comput. 24(1), 445-460 (2020). https://doi.org/10.1007/s00500-01903924-5

27. Wang, X., Xu, H., Zhang, S., Liang, L., Niu, P., Yang, H.: A color image watermarking approach based on synchronization correction. Fundam. Inf. 158(4), 385-407 (2018). https://doi.org/ 10.3233/FI-2018-1654

28. Zheng, Q., Liu, N., Wang, F.: An Adaptive embedding strength watermarking algorithm based on shearlets' capture directional features. Mathematics 8(8), 1377 (2020). https://doi.org/10.3390/ math8081377

29. Favorskaya, M.N., Jain, L.C., Savchina, E.I.: Perceptually tuned watermarking using non-subsampled shearlet transform. In: Computer Vision in Control Systems-4: Real Life Applications, pp. 41-69. Intelligent Systems Reference Library. Springer, Cham (2018). https://doi.org/10.1007/978-3-319-67994-5_3

30. Chen, C., Liu, J., Sun, J., Ren, Z., Hu, H.: A robust watermarking scheme based on non-subsampled contourlet transform. In: 2010 8th World Congress on Intelligent Control and Automation, pp. 1022-1026 (2010). https://doi.org/10.1109/WCICA.2010. 5554703

31. Gao, G., Cai, B., Xu, S., Yan, T.: Watermark performance contrast between contourlet and non-subsampled contourlet transform. In: 2012 IEEE International Conference on Information and Automation, pp. 507-511 (2012). https://doi.org/10.1109/ICInfA. 2012.6246859

32. Hu, H.-T., Chang, J.-R., Hsu, L.-Y.: Robust blind image watermarking by modulating the mean of partly sign-altered DCT coefficients guided by human visual perception. AEU Int. J. Electron. Commun. 70(10), 1374-1381 (2016). https://doi.org/ 10.1016/j.aeue.2016.07.011

33. Moosazadeh, M., Ekbatanifard, G.: A new DCT-based robust image watermarking method using teaching-learning-based optimization. J. Inf. Security Appl. 47, 28-38 (2019). https://doi. org/10.1016/j.jisa.2019.04.001

34. Assini, I., Badri, A., Safi, K., Sahel, A., Baghdad, A.: A robust hybrid watermarking technique for securing medical image. Int.
J. Intell. Eng. Syst. (2018). https://doi.org/10.22266/IJIES2018. 0630.18

35. Verma, V., Sharma, N.: Hybrid mode of medical image watermarking to enhance robustness and imperceptibility. Int. J. Innov. Technol. Explor. Eng. 9, 351-359 (2020)

36. Anand, A., Singh, A.K.: An improved DWT-SVD domain watermarking for medical information security. Comput. Commun. 152, 72-80 (2020). https://doi.org/10.1016/j.comcom.2020. 01.038

37. Sankaran, K.S., Abhi Rayna, H., Mangu, V., Prakash, V.R., Vasudevan, N.: Image water marking using DWT to encapsulate data in medical image. In: 2019 International Conference on Communication and Signal Processing (ICCSP) (2019). https:// doi.org/10.1109/ICCSP.2019.8698057.

38. Alshanbari, H.S.: Medical image watermarking for ownership \& tamper detection. Multimedia Tools Appl. (2020). https://doi.org/ 10.1007/s11042-020-08814-9

39. Devi, H.S., Singh, K.M.: Red-Cyan anaglyph image watermarking using DWT, Hadamard transform and singular value decomposition for copyright protection. J. Inf. Security Appl. 50, 102424 (2020). https://doi.org/10.1016/j.jisa.2019.102424

40. Guo, A., Meihua, Xu., Ran, F., Wang, H.: A novel medical internet of things perception system based on visual image encryption and intrusion detection. Clust. Comput. 22, 13405-13413 (2019). https://doi.org/10.1007/s10586-018-1944-2

41. Khaldi, A.: A lossless blind image data hiding scheme for semifragile image watermark. Int. J. Comput. Vis. Robot. 10(5), 373 (2020). https://doi.org/10.1504/IJCVR.2020.109389

42. Xie, J., Wu, D., Wang, J., Liu, C.: The analysis of additive noise of thermal test low-frequency cable fault detection based on M sequence correlation algorithm. Clust. Comput. 22, 9303-9312 (2019). https://doi.org/10.1007/s10586-018-2130-

Publisher's Note Springer Nature remains neutral with regard to jurisdictional claims in published maps and institutional affiliations.

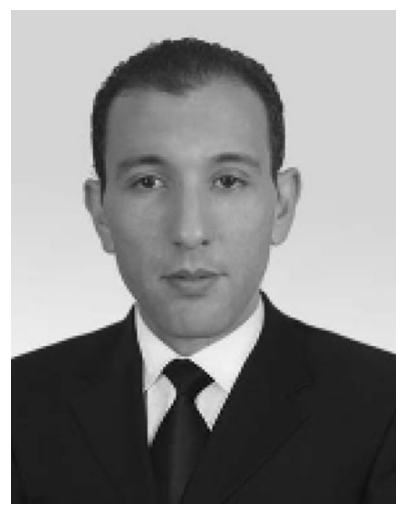

Fares Kahlessenane currently works as Assistant Professor at the Faculty of New Technologies of Information and Communication (FNTIC), University Kasdi Merbah Ouargla. His researches focus on digital Watermarking, signal and image processing and audio watermarking. 


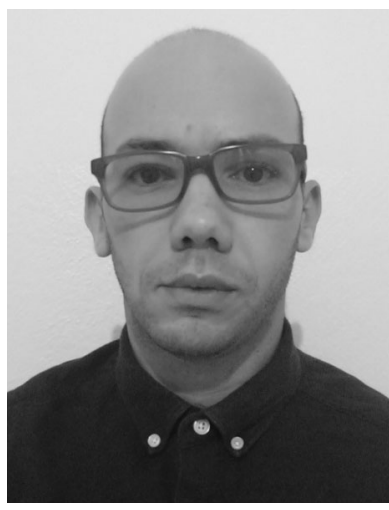

insertion domain.

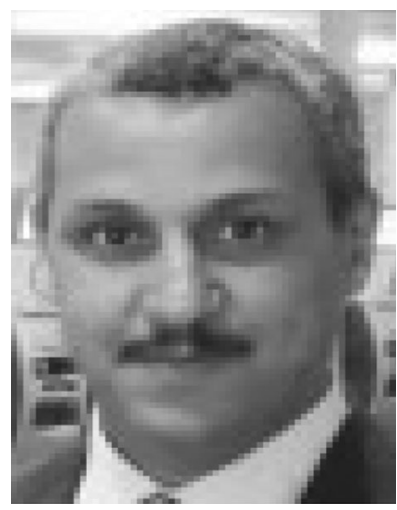

Amine Khaldi currently works as Assistant Professor at the Faculty of New Technologies of Information and Communication (FNTIC), University Kasdi Merbah Ouargla. His researches focus on digital Watermarking, Cryptography, Image Steganography, Data Hiding and Network Security. He also worked on Image Segmentation, Pattern Recognition and Range Image Processing. His current watermarking research projects focus on the spatial and frequency

Redouane Kafi currently works at the Department of Electrical Engineering, Université Kasdi Merbah Ouargla. Kafi does research in Aeronautical Engineering, Control Systems Engineering and Electronic Engineering.

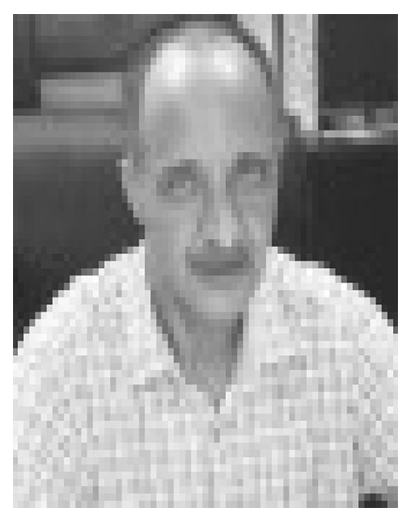

Salah Euschi currently works at the Department of Computer science, Université Kasdi Merbah Ouargla. Euschi does research in Cryptography, Image Steganography, Data Hiding and Network Security and Electronic Engineering. 